\title{
Synergistic Inhibition of Glioma Cell Proliferation by Withaferin A and Tumor Treating Fields
}

\author{
Edwin Chang ${ }^{1}$, Christoph Pohling ${ }^{1}$, Nooshin Beygui ${ }^{2}$, Chirag B. Patel ${ }^{1}$, Jarrett Rosenberg ${ }^{1}$, \\ Dong $\mathrm{Ho} \mathrm{Ha}^{1}$, and Sanjiv S. Gambhir ${ }^{1}$ \\ ${ }^{1}$ Department of Radiology, Molecular Imaging Program at Stanford, Canary Center for Early \\ Cancer Detection, Stanford University, Palo Alto, California, USA \\ ${ }^{2}$ Scripps College, Claremont, California, USA
}

\begin{abstract}
BACKGROUND—Glioblastoma (GBM) is the most aggressive and lethal form of brain cancer. Standard therapies are non-specific and often of limited effectiveness; thus, efforts are underway to uncover novel, unorthodox therapies against GBM. In previous studies, we investigated Withaferin A, a steroidal lactone from Ayurvedic medicine that inhibits proliferation in cancers including GBM. Another novel approach, tumor treating fields (TTFields), is thought to disrupt mitotic spindle formation and stymie proliferation of actively dividing cells. We hypothesized that combining TTFields with Withaferin A would synergistically inhibit proliferation in glioblastoma.
\end{abstract}

METHODS-Human glioblastoma cells (GBM2, GBM39, U87-MG) and human breast adenocarcinoma cells (MDA-MB-231) were isolated from primary tumors. The glioma cell lines were genetically engineered to express firefly luciferase. Proliferative potential was assessed either by bioluminescence imaging or cell counting via hemocytometer.

RESULTS-TTFields (4 V/cm) significantly inhibited growth of the four cancer cell lines tested $(\mathrm{n}=3$ experiments per time point, 4 measurements per sample, $\mathrm{p}<0.02$ at least; 2 -way ANOVA, control vs. treatment). The combination of Withaferin A (10-100 nM) with TTFields significantly inhibited the growth of the glioma cells to a degree beyond that of Withaferin A or TTFields alone. The interaction of the Withaferin A and TTFields on glioma cells was found to be synergistic in nature ( $\mathrm{p}<0.01, \mathrm{n}=3$ experiments). These findings were validated by both bioluminescence and hemocytometric measurements.

CONCLUSIONS-The combination of Withaferin A with TTFields represents a novel approach to treat GBM in a manner that is likely better than either treatment alone and that is synergistic.

\section{Keywords}

Alternating electric fields; combination therapy; glioblastoma; synergy; tumor treating fields; Withaferin A

\footnotetext{
Please send correspondences to: Sanjiv S. Gambhir, MD, PhD, Virginia and D. K. Ludwig Professor, Chairman, Departments of Radiology and Bioengineering, Director, Molecular Imaging Program at Stanford, Director, Canary Center at Stanford for Cancer Early Detection, Head, Nuclear Medicine, Phone: 650-725-2309, Fax: 650-724-4948, sgambhir@stanford.edu.

Conflict of Interest: The authors declare no conflict of interest and agree on the submission of this manuscripts
} 


\section{Introduction}

Glioblastoma (GBM) is the most common yet deadliest form of primary brain cancer (median survival time: 15-17 months; 5-year survival: 5-10\% [1, 2]. Given its aggressive nature, current interventions consist of equally aggressive surgical resection and subsequent radio- and chemotherapy. Because the cancer is highly heterogeneous, these standardized but non-specific therapies are often ineffective [2]. To establish progress against GBM, new, unorthodox therapies must be pursued.

Treatment of cancers by tumor treating fields (TTFields) is a novel, validated therapy that may represent an additional paradigm in anti-cancer treatments [3]. While not definitively established, the mechanism of anti-cancer action by TTFields most likely rests upon the property that actin filaments have intrinsic dipole moments [4]. By forcing actin filaments to align along electric field lines through the imposition of a TTField, one interrupts the functionality of the mitotic spindle in actively dividing cells[5], thereby disrupting the actinbased cytoskeleton supporting the mitotic spindles [4]. Such stress on the spindles perturbs cellular division [4]. Numerous proof of concept experiments as well as relevant technological developments have occurred over the last ten years [4], culminating in the approval by the Food and Drug Administration (FDA) of a commercial, clinical TTField device (Optune ${ }^{\circledR}$, Novocure Ltd., Jersey, UK) for the treatment of newly diagnosed glioblastoma [6].

There have been clinical investigations into combination therapies. Treatment by TTFields in combination with temozolomide has resulted in significant extension of overall survival in glioblastoma patients [7]. Cases of combination therapy of TTFields with bevacizumab have been reported [8]. Preliminary findings suggests that the interaction between TTField and either temozolomide or bevacizumab are additive as opposed to synergistic but the data requires further elucidation. For detailed mathematical definition of additivity versus synergy, please see Fang et al. [9].

Withaferin A is a steroidal lactone that originates from the roots and leaves of the winter cherry plant (Withania somnifera) [10]. Recently, we demonstrated, for the first time, the effectiveness of Withaferin A in inhibiting the growth of human gliomas in cell culture and in preclinical murine models of orthotopic GBM [11]. A unified insight into Withaferin A's action is still forthcoming, but researchers have shown it to affect expression and activity of numerous transcription factors such as NF- $\kappa \mathrm{B}$ and Sp1 [12, 13]. Like TTFields, these factors impact the dynamics of cytoskeletal assembly and disassembly [14-16]. Thus, it is unsurprising that Withaferin $\mathrm{A}$ is associated with disruption of vimentin/actin assembly and cytoskeletal organization [17-19]. We thus hypothesized that the combination of TTFields and Withaferin A, the main component of an Ayurvedic natural product, retards glioblastoma proliferation in a synergistic manner. We report here the effects of the combination therapy approach on three glioblastoma cell lines: U87-MG, GBM2 and GBM39. All are human-derived and have functional, morphological and genomic variations that represent the heterogeneity of gliomas. The current investigation builds upon a previous study [11] in which we looked at the role of natural products in advancing anti-glioblastoma therapies. 


\section{Material and Methods}

\section{Cell Culture Studies}

Two patient-derived GBM lines (GBM2 [20], GBM39 [21]) and one commercially available human GBM cell line (U87-MG from ATCC, Manassas, VA, USA) were used for our studies. The breast adenocarcinoma cell line, MDA-MB-231, was also purchased from ATCC. GBM2 originated from the Stanford University Medical Hospital and was obtained for research purposes after approval from the University's Internal Review Board (IRB-28612). GBM39 was a gift from Dr. Paul Mischel (Ludwig Institute for Cancer Research, University of California at San Diego). Details of cell culture conditions summarized in Supplemental Method One

\section{Seeding of Cells onto Thermanox Cover Slips for TTField Experiments}

Approximately 50,000 single cells were seeded onto the center of a $22 \mathrm{~mm}$ diameter glass or plastic Nunc Thermanox ${ }^{\mathrm{TM}}$ cover slip (ThermoFisher Scientific, Waltham, MA, USA) in 6well plates. The cells were left to grow on the cover slip for two to three days before being transferred to a ceramic dish of an inovitro ${ }^{\mathrm{TM}}$ in vitro TTField device (Novocure Inc., Haifa, Israel). Refer to Supplemental Method Two.

\section{In vitro Tumor Treating Field Device}

A workflow for a typical TTField experiment is summarized in Figure 1. Details described in Figure 1 legend. Cells were grown in the ceramic dishes of the inovitro ${ }^{\text {TM }}$ system for 3-6 days and then trypsinized and counted according to standardized protocols. Refer to Supplemental Method Two for details.

\section{Cell Counting Assay via Hemocytometer}

Cell counting methodologies and averaging were achieved by standardized protocols and visualized on a Zeiss PrimoVert benchtop microscope (Dublin, CA, USA). Unless otherwise stated, cell counts were done on trypsinized, single-cell suspensions with a hemocytometer and the median of the four cell-count measurements was calculated and rounded to the nearest integer. Please refer to legend of Supplemental Figure S1 for details

\section{Bioluminescence Imaging}

For all bioluminescence work, we used genetically-modified GBM2, GBM39 and U87-MG whereby cells were transfected with lentiviral vectors that expressed either firefly luciferase (for GBM39) or a fusion protein of GFP and firefly luciferase (for GBM2 and U87) [22, 23]. All BLI work done on an IVIS Spectrum (Xenogen Corporation, Alameda, CA). Please refer to legend of Supplemental Figure S2 for details

\section{Purchase of Chemical Reagents}

Unless otherwise stated, all chemicals, including Withaferin A, were purchased either from Selleckchem Inc. (Houston, TX, USA) or Sigma-Aldrich (St. Louis, MO, USA). 


\section{Statistical Analysis}

Treatments of TTFields vs. Withaferin A alone as well as combination treatments of TTFields and Withaferin A were assessed for significance of effect through either 2-way analysis of variance (ANOVA) or significance analysis of regression [9, 24]. Generally, 2way ANOVA's were used to determine synergy of two treatments at fixed concentration for each treatment. Significance analysis of regression was used if one was examining a dose range for one or both treatments. A Poisson regression was then done with factors of $\log _{10}$ (Withaferin dose), TTFields (on at $4 \mathrm{~V} / \mathrm{cm}$ vs. off), and their interaction. 2-way ANOVA was performed using the PRISM 7.0 application (GraphPad Software Inc., La Jolla, CA, USA) while Poisson-based regression analysis was completed with the assistance of two biostatisticians. Bonferroni or Dunnet post-hoc corrections were employed to adjust alpha for multiple comparisons.

\section{Modeling of Electric Fields}

Modeling of electric fields in the well of a hypothetical ceramic dish was completed with Comsol 4.2 Multiphysics simulation software (Comsol Multiphysics Inc., Burlington, MA).

\section{Results}

TTFields decrease cellular growth and increased sensitivity to doxorubicin in MDA-MB-231 cells

To investigate if the TTField effect could be reproduced between labs [25], we examined the effect of TTFields (frequency $150 \mathrm{kHz}$ ) on growth patterns of a breast carcinoma cell line (MDA-MB-231). Cellular counts were significantly reduced ( $n=3$ measurements per time point, $\mathrm{p}<0.001$ ) when compared to MDA-MB-231 cells grown without the presence of TTFields (Supplemental Figure S1A).

To examine if an applied TTField could sensitize cancer cells to chemotherapeutic agents, we did a dose response study of MDA-MB-231 cellular growth to escalating concentrations of doxorubicin (Supplemental Figure S1B) in the absence of TTFields where an $\mathrm{IC}_{50}$ of 0.31 $\mu \mathrm{M}$ for doxorubicin was determined. The application of TTFields $(4 \mathrm{~V} / \mathrm{cm}, 200 \mathrm{kHz})$ consistently decreased MDA-MB-231 cell number for all concentrations of doxorubicin that were studied. These studies verified previous findings [25] and were important to do prior to moving on to studies with GBM cell lines

\section{Reduced cell proliferation due to TTFields is apparent in several human-derived glioma cell lines}

The study shown in Supplemental Figure S1 was extended to three different human-derived, glioma cell lines: GBM2, GBM39 and U87-MG. For all three cell lines, the application of a TTField $(4 \mathrm{~V} / \mathrm{cm})$ led to a progressive and significant decline $(\mathrm{p}<0.0001$, TTField vs control for GBM2 and U87-MG; $\mathrm{p}<0.02$ for GBM39; $\mathrm{n}=3$ samples per time point for all) in cellular growth (Figure 2A-C) when compared to non-TTField controls. The largest differences occurred with U87-MG. 


\section{Synergistic inhibition of glioma proliferation by Withaferin A and TTFields}

Withaferin A has been shown to inhibit the growth of several glioma lines at an $\mathrm{IC}_{50}$ of 250 $\mathrm{nM}$ [11]. When U87-MG cells were exposed to $0.1 \mu \mathrm{M}$ of Withaferin A, no significant loss of cell number (with respect to no treatment controls) was observed at three days (Figure $3 \mathrm{~A}$ ) or six days (Figure $3 \mathrm{~B}$ ) post treatment. Addition of an alternating electric field $(4 \mathrm{~V} / \mathrm{cm}$ or $2.5 \mathrm{~V} / \mathrm{cm} ; 3$ days and 6 days, respectively) led to significant reduction (at least $\mathrm{p}<0.02$, TTField vs control, $n=3$ samples) in cell number for the TTField samples (Figure 3A-B). Addition of $0.1 \mu \mathrm{M}$ Withaferin A to TTFields led to a further, significant decrease in cell number with respect to TTField alone ( $\mathrm{p}<0.0001$, TTField vs TTField + Withaferin A, $n=3$ samples). These findings provide statistical evidence that there was a significant, synergistic interaction between the treatments of TTField ( $0 \%$ reduction alone) and Withaferin A ( $25 \%$ reduction alone) on blocking the proliferation of actively growing, human-derived glioma cells (55\% reduction in combination).

We extended the interaction studies between Withaferin A and TTFields to the patientderived glioma cell lines of U87-MG, GBM39 and GBM2 [11] (Figure 3C-E). Also, we examined the effect of several concentrations of Withaferin A (from $0 \mu \mathrm{M}$ to $0.3 \mu \mathrm{M}$ of drug), in the absence and presence of $4 \mathrm{~V} / \mathrm{cm}$ TTFields at $200 \mathrm{kHz}$. In all three human glioma cell lines (U87-MG, GBM39, GBM2, Figure 3C-E), we found that, in the presence of TTFields, cell culture growth was significantly impeded ( $\mathrm{p}<0.001, \mathrm{n}=3$ samples, TTField vs no TTField) at all doses of Withaferin A tested except for $0 \mu \mathrm{M}$ (data point on the y-axis). These Poisson-based Analysis of Regression findings of statistically different dose response trends between the absence and the presence of TTFields demonstrates synergistic interactivity between the TTFields and Withaferin A treatments.

Comparative bright field pictographs of corresponding cells further validate the synergy between the two treatments (Figure 3F). In the case of treatment with $0.1 \mu \mathrm{M}$ Withaferin A alone, there was no qualitative difference in the number and size of adherent neurospheres, nor of the patterns in the connective network of the surrounding adherent cells. The application of $200 \mathrm{kHz}$ of TTField alone seemed to disrupt the connective network of the adherent cells around the neurospheres. With the combination of TTFields $(4 \mathrm{~V} / \mathrm{cm})$ and Withaferin $\mathrm{A}(0.1 \mu \mathrm{M})$, there was a more extensive disruption of the connected network of adherent cells as well as a diminution of size and number of adherent neurospheres. When U87-MG/eGFP-Luc cell's bioluminescence imaging (BLI) activity was assessed with respect to challenge by TTFields $(4 \mathrm{~V} / \mathrm{cm}, 200 \mathrm{kHz})$, Withaferin $\mathrm{A}(0.1 \mu \mathrm{M})$, or both treatments, it was found that the combination of Withaferin A and TTFields reduced BLI activity (with respect to no Withaferin A controls) by $83 \%$ (100\% vs $16.9 \%$, TTFields alone compared to TTFields+Withaferin A) while in conditions with no TTFields, BLI activity was reduced by only $49.1 \%$ (Supplemental Figures S2A-B). A parallel study on GBM39/luc cells also yielded similar bright-field and bioluminescence observations (Supplemental Figures S2C-D).

\section{Spatial temperature differential of ceramic dishes does not significantly affect cell growth}

Comsol modeling (Supplemental Figure S3A) and temperature probing showed that the center of the wells had a mean temperature of $37.6 \pm 0.4^{\circ} \mathrm{C}$ while the periphery had a 
significantly ( $<<0.00001$, paired t-test) lower temperature of $36.7 \pm 0.4^{\circ} \mathrm{C}$ (Supplemental Figure S3B). U87-MG cells incubated at $39^{\circ} \mathrm{C}$ (but in the absence of a TTField) showed no difference in proliferation when compared to cells incubated at $37^{\circ} \mathrm{C}$ (Supplemental Figure $\mathrm{S} 3 \mathrm{C})$.

\section{Effect of TTField frequency on U87-MG's cell count and sensitivity to Withaferin A}

To examine if phenomena other than the TTField are responsible for decreased cell numbers as well as enhanced sensitivity to Withaferin A, U87-MG cells were exposed to different frequencies in TTFields from $50 \mathrm{kHz}$ to $500 \mathrm{kHz}$ and compared to U87-MG cells not subjected to TTFields (Figure 4A). TTField-exposed cells showed no significant difference in cellular population compared to control cells for frequencies of 50, 100 and $500 \mathrm{kHz}$. However, U87-MG cell numbers were significantly reduced at frequencies of 200 and 400 $\mathrm{kHz}$ ( $\mathrm{p}<0.0001$, one-way ANOVA, multiple comparison with respect to no TTField controls, Bonferroni and Dunnett post-hoc corrections). These findings are consistent with findings in the literature for other cancer cell types [4, 26].

At $50 \mathrm{kHz}$ TTFields, U87-MG cells showed no enhanced sensitivity to Withaferin A (dose range: $0-0.3 \mu \mathrm{M}$, Figure 4B). In contrast, when lower concentrations of Withaferin A (dose range $0-0.1 \mu \mathrm{M}$ ) were combined with $200 \mathrm{kHz}$ TTFields, cell proliferation was significantly reduced further ( $\mathrm{p}<0.0001$, Figure 4C). Previously, it was shown that TTFields alone at this frequency are effective in reducing cell number (Figure 4A), as well as being synergistic against U87-MG cell proliferation when combined with a broader range of Withaferin A doses (Figure 3C). Taken together, the aforementioned findings would suggest that there is some interaction between the two treatment approaches to enhance U87-MG's susceptibility to lower doses of Withaferin A. At $500 \mathrm{kHz}$, where neither Withaferin A nor TTFields alone do not significantly affect cell growth (Figure 4A), the application of the alternating field at this frequency did significantly ( $\mathrm{p}<0.0001$ ) sensitize U87-MG cells to low doses of Withaferin A (Figure 4D).

\section{Discussion}

Our manuscript is the first report on synergistic interaction in therapy between TTFields with the natural product, Withaferin A. TTField application can sensitize glioblastoma stem cells from temozolomide-resistant patients towards temozolomide therapy [7, 27]. Several different TTField combination therapies with numerous cytostatic agents (metformin, mefloquine, ganciclovir, bumetanide, and minocycline) have been studied in glioma cell lines but in no treatment regimen was there more than an additive improvement in efficacy of the cytostatic [28] while statistical analysis in the current study indicated therapeutic synergy with co-treatments of Withaferin A and TTFields.

It is known, that TTFields can stall replication of actively growing cells by disruption of cytoskeletal proteins, such as septin, that participate in the stabilization of the mitotic spindle of dividing cells [29]. Though the mechanisms of action for Withaferin A are also not fully elucidated, there have been reports suggesting that the lactonal steroid acts through disruption of the cytoskeletal framework as well $[18,19]$. However more experiments will be needed to determine if this is indeed the reason for the synergistic interaction between 
Withaferin A and TTFields. Interestingly, combination studies with other mitotic spindle disruptors such as chloroquine [30] or IN-3, [31] revealed data suggesting synergistic, TTField-mediated disruption in glioma proliferation that acts either through multiple disruptions along the mitotic spindle or through several sites of interference throughout the cytoskeletal network supporting the spindle or through both.

The FDA has approved the clinical use of TTFields in newly-diagnosed GBM patients [6] with the Optune ${ }^{\circledR}$ TTField device [5]. Such acceptance of therapy has prompted several lines of investigation into the combination of TTFields with established standard of care approaches such as temozolomide [7, 27], bevacizumab [8, 32, 33], and ionizing radiation [34]. The novel combination of TTFields with Withaferin A commands interest because of their synergistic interaction against human-derived glioma cell cultures. However, more preclinical and clinical work will be necessary to establish if the synergistic interaction between the treatments of TTFields and Withaferin A exists in murine models and in GBM patients respectively. Unfortunately, such deeper investigations are hampered by the current unavailability of a functional device to produce TTFields on preclinical, murine and orthotopic models of GBM.

Unlike TTFields, Withaferin A and related withanolides have yet to be approved by the FDA as anti-cancer agents. However, withanolides are effective against different cell culture strains of cancers [35] as well as in numerous preclinical models of murine and human cancer xenografts [36], including human GBM [11]. Such investigations have also included toxicity studies in preclinical models $[37,38]$ and in clinical subjects $[39,40]$. In general, these studies showed good tolerability to oral doses of either purified Withaferin A or Withania extracts that contain withanolides with minimal side effects Future preclinical and clinical investigations should include investigating the impact of metabolism on efficacy of Withaferin A [35]. The addition of such studies would likely bring approval of Withaferin A as an agent against cancer.

In the set-up of our studies we could not entirely separate the effects of heat generation and alternating electric fields on glioma cell viability. Nevertheless, in a rabbit study of TTFields in vivo, tumors treated for 21 days with sham electrodes heated to mimic temperatures generated by the TTFields $\left(38-39.9^{\circ} \mathrm{C}\right)$ increased in volume by a factor of 70 from baseline, whereas tumors treated with TTFields grew by a factor of 34 from baseline [25]. We showed that there was no difference in U87-MG cell growth for eight days between temperatures of $37^{\circ} \mathrm{C}$ and $39^{\circ} \mathrm{C}$ (Supplemental Figure S3C). Finally, hyperthermia, used to treat malignant gliomas either as a single treatment or in combination with radiotherapy [41], is effective between $42^{\circ} \mathrm{C}$ to $43^{\circ} \mathrm{C}$, i.e., beyond the hyperthermic conditions explored in our experiments. These reports, in aggregate, would suggest that synergistic effects reported in this study are due to TTField and Withaferin A and not confounded by the presence of heat generation.

Another promising avenue of treatment is immunotherapy against glioblastomas [42]. Withaferin $\mathrm{A}$ is a steroid that has been shown to inhibit $\mathrm{T}$ cell-mediated immune response [43]. On the other hand, the scientific literature indicates that Withaferin A can display both immuno-inhibitory and immuno-stimulatory properties [35]. TTFields can affect $\mathrm{T}$ cells by 
partially inhibiting proliferation; however, TTFields do not impair any pivotal functional properties of the surviving T cells [44]. In addition, the application of TTFields on MDAMB-231 breast cancer cells prompted the line to express immune stimulatory stress marker proteins such as calreticulin and HSP70 as well as the secretion of HMGB1 [45]. Thus currently, the impact of combination therapy by TTFields and Withaferin A on the immune system (and consequently on immunotherapy) is undefined. A beneficial impact will most likely depend upon uncovering optimal field strengths and frequencies for the TTFields as well as appropriate dosage windows for Withaferin A that would minimally influence immune function.

In summary, the combination of Withaferin A with TTFields represents a novel approach to treat GBM. Given the aggressiveness of the disease as well as the paucity of the diagnostic and therapeutic tools arrayed against GBM, such a unique approach is worthy of further pursuit.

\section{Supplementary Material}

Refer to Web version on PubMed Central for supplementary material.

\section{Acknowledgments}

Funding Sources: Ben and Catherine Ivy Foundation (SSG) and the R25 Translational Neuroscience Training Grant R25NS065741 (CBP)

We acknowledge the excellent technical support of Kathryn Li, Tara Thakurta, Alex Sera ni and Xiaofan Wu. We gratefully acknowledge the Ben and Catherine Ivy Foundation (SSG) and the R25 Translational Neuroscience Training Grant R25NS065741 (CBP) for their support of our research. We thank Novocure Inc. for providing us with the inovitro ${ }^{\mathrm{TM}}$ device as well as technical support. Finally, we thank Dr. Moshe Giladi for all of his thoughts, comments and feedback.

\section{List of Abbreviations}

$\begin{array}{ll}\text { ANOVA } & \text { Analysis of Variance } \\ \text { BLI } & \text { Bioluminescence Imaging } \\ \text { DAPI } & 4^{\prime}, 6 \text {-Diamidino-2-Phenylindole } \\ \text { DMEM } & \text { Dulbecco's Modified Eagle's Medium } \\ \text { H-EGF } & \text { Human Epidermal Growth Factor } \\ \text { EGFR } & \text { Epidermal Growth Factor Receptor } \\ \text { EGFRvIII } & \text { Epidermal Growth Factor Receptor variant III } \\ \text { FBS } & \text { Fetal Bovine Serum } \\ \text { FDA } & \text { Food and Drug Administration } \\ \text { H-FGF } & \text { Human Fibroblast Growth Factor } \\ \text { GBM } & \text { Glioblastoma }\end{array}$


GBM2

GBM2/GFP-Luc

GBM39

GBM39/Luc

GFP

GFP/Luc

MDA-MB-231

PBS

H-PDGF-AA

H-PDGF-BB

TTField

U87-MG

U87-MG/eGFP-Luc
Patient-derived glioblastoma cell culture (from Stanford University School of Medicine)

GBM2 that was genetically modified to express a fusion protein of firefly luciferase and GFP

Patient-derived glioblastoma cell culture (from University of California at San Diego School of Medicine)

GBM39 that was genetically modified to express firefly luciferase

Green Fluorescent Protein

Fusion protein of GFP and firefly luciferase

Human breast adenocarcinoma cancer cell line (from ATCC)

Phosphate-buffered Saline

Human Platelet-derived Growth Factor variant AA

Human Platelet-derived Growth Factor variant BB

Tumor Treating Field

Human-derived GBM cell line that was purchased from ATCC

U87-MG that was genetically modified to express a fusion protein of firefly luciferase and GFP

\section{References}

1. Stupp R, Hegi ME, Mason WP, van den Bent MJ, Taphoorn MJ, Janzer RC, Ludwin SK, Allgeier A, Fisher B, Belanger K, Hau P, Brandes AA, Gijtenbeek J, Marosi C, Vecht CJ, Mokhtari K, Wesseling P, Villa S, Eisenhauer E, Gorlia T, Weller M, Lacombe D, Cairncross JG, Mirimanoff RO. European Organisation for R, Treatment of Cancer Brain T, Radiation Oncology G, National Cancer Institute of Canada Clinical Trials G. Effects of radiotherapy with concomitant and adjuvant temozolomide versus radiotherapy alone on survival in glioblastoma in a randomised phase III study: 5-year analysis of the EORTC-NCIC trial. Lancet Oncol. 2009; 10:459-466. DOI: 10.1016/ S1470-2045(09)70025-7 [PubMed: 19269895]

2. Johnson DR, O'Neill BP. Glioblastoma survival in the United States before and during the temozolomide era. J Neurooncol. 2012; 107:359-364. DOI: 10.1007/s11060-011-0749-4 [PubMed: 22045118]

3. Davies AM, Weinberg U, Palti Y. Tumor treating fields: a new frontier in cancer therapy. Ann N Y Acad Sci. 2013; 1291:86-95. DOI: 10.1111/nyas.12112 [PubMed: 23659608]

4. Giladi M, Schneiderman RS, Voloshin T, Porat Y, Munster M, Blat R, Sherbo S, Bomzon Z, Urman N, Itzhaki A, Cahal S, Shteingauz A, Chaudhry A, Kirson ED, Weinberg U, Palti Y. Mitotic Spindle Disruption by Alternating Electric Fields Leads to Improper Chromosome Segregation and Mitotic Catastrophe in Cancer Cells. Scientific Reports. 2015; 5:18046.doi: 10.1038/srep18046 [PubMed: 26658786] 
5. Hottinger AF, Pacheco P, Stupp R. Tumor treating fields: a novel treatment modality and its use in brain tumors. Neuro Oncol. 2016; 18:1338-1349. DOI: 10.1093/neuonc/now182 [PubMed: 27664860]

6. Chaudhry A, Benson L, Varshaver M, Farber O, Weinberg U, Kirson E, Palti Y. NovoTTF-100A System (Tumor Treating Fields) transducer array layout planning for glioblastoma: a NovoTAL system user study. World J Surg Oncol. 2015; 13:316.doi: 10.1186/s12957-015-0722-3 [PubMed: 26558989]

7. Stupp R, Taillibert S, Kanner AA, Kesari S, Steinberg DM, Toms SA, Taylor LP, Lieberman F, Silvani A, Fink KL, Barnett GH, Zhu JJ, Henson JW, Engelhard HH, Chen TC, Tran DD, Sroubek J, Tran ND, Hottinger AF, Landolfi J, Desai R, Caroli M, Kew Y, Honnorat J, Idbaih A, Kirson ED, Weinberg U, Palti Y, Hegi ME, Ram Z. Maintenance Therapy With Tumor-Treating Fields Plus Temozolomide vs Temozolomide Alone for Glioblastoma: A Randomized Clinical Trial. Jama. 2015; 314:2535-2543. DOI: 10.1001/JAMA.2015.16669 [PubMed: 26670971]

8. Ansstas G, Tran DD. Treatment with Tumor-Treating Fields Therapy and Pulse Dose Bevacizumab in Patients with Bevacizumab-Refractory Recurrent Glioblastoma: A Case Series. Case Rep Neurol. 2016; 8:1-9. DOI: 10.1159/000442196 [PubMed: 26889149]

9. Fang HB, Ross DD, Sausville E, Tan M. Experimental design and interaction analysis of combination studies of drugs with log-linear dose responses. Stat Med. 2008; 27:3071-3083. DOI: 10.1002/sim.3204 [PubMed: 18186545]

10. Lavie D, Glotter E, Shvo Y. Constituents of Withania somnifera Dun. III. The side chain of Withaferin A. Journal of Organic Chemistry. 1965; 30:1774-1778.

11. Chang E, Pohling C, Natarajan A, Witney TH, Kaur J, Xu L, Gowrishankar G, ALDS, Murty S, Schick S, Chen L, Wu N, Khaw P, Mischel P, Abbasi T, Usmani S, Mallick P, Gambhir SS. AshwaMAX and Withaferin A inhibits gliomas in cellular and murine orthotopic models. $\mathbf{J}$ Neurooncol. 2016; 126:253-264. DOI: 10.1007/s11060-015-1972-1 [PubMed: 26650066]

12. Prasanna KS, Shilpa P, Salimath BP. Withaferin A suppresses the expression of vascular endothelial growth factor in Ehrlich ascites tumor cells via Sp1 transcription factor. Current Trends in Biotechnology and Pharmacy. 2009; 3:138-148.

13. Heyninck K, Lahtela-Kakkonen M, Van der Veken P, Haegeman G, Vanden Berghe W. Withaferin A inhibits NF-kappaB activation by targeting cysteine 179 in IKKbeta. Biochem Pharmacol. 2014; 91:501-509. DOI: 10.1016/j.bcp.2014.08.004 [PubMed: 25159986]

14. Zhou C, Ji J, Cai Q, Shi M, Chen X, Yu Y, Liu B, Zhu Z, Zhang J. MTA2 promotes gastric cancer cells invasion and is transcriptionally regulated by Sp1. Mol Cancer. 2013; 12:102.doi: 10.1186/1476-4598-12-102 [PubMed: 24010737]

15. Kustermans G, El Benna J, Piette J, Legrand-Poels S. Perturbation of actin dynamics induces NFkappaB activation in myelomonocytic cells through an NADPH oxidase-dependent pathway. Biochem J. 2005; 387:531-540. DOI: 10.1042/BJ20041318 [PubMed: 15535802]

16. Nemeth ZH, Deitch EA, Davidson MT, Szabo C, Vizi ES, Hasko G. Disruption of the actin cytoskeleton results in nuclear factor-kappaB activation and inflammatory mediator production in cultured human intestinal epithelial cells. J Cell Physiol. 2004; 200:71-81. DOI: 10.1002/jcp. 10477 [PubMed: 15137059]

17. Bargagna-Mohan P. Small Molecule Anti-Angiogenic Probes of the Ubiquitin Proteasome Pathway: Potential Application to Choroidal Neovascularization. Invest Ophthalmol Vis Sci. 2006; 47:4138-4145. DOI: 10.1167/iovs.05-1452 [PubMed: 16936134]

18. Satelli A, Li S. Vimentin in cancer and its potential as a molecular target for cancer therapy. Cell Mol Life Sci. 2011; 68:3033-3046. DOI: 10.1007/s00018-011-0735-1 [PubMed: 21637948]

19. Bargagna-Mohan P, Hamza A, Kim YE, Khuan Abby Ho Y, Mor-Vaknin N, Wendschlag N, Liu J, Evans RM, Markovitz DM, Zhan CG, Kim KB, Mohan R. The tumor inhibitor and antiangiogenic agent withaferin A targets the intermediate filament protein vimentin. Chem Biol. 2007; 14:623634. DOI: 10.1016/j.chembiol.2007.04.010 [PubMed: 17584610]

20. Grasso CS, Tang Y, Truffaux N, Berlow NE, Liu L, Debily MA, Quist MJ, Davis LE, Huang EC, Woo PJ, Ponnuswami A, Chen S, Johung TB, Sun W, Kogiso M, Du Y, Qi L, Huang Y, HuttCabezas M, Warren KE, Le Dret L, Meltzer PS, Mao H, Quezado M, van Vuurden DG, Abraham J, Fouladi M, Svalina MN, Wang N, Hawkins C, Nazarian J, Alonso MM, Raabe EH, Hulleman E, 
Spellman PT, Li XN, Keller C, Pal R, Grill J, Monje M. Functionally defined therapeutic targets in diffuse intrinsic pontine glioma. Nat Med. 2015; doi: 10.1038/nm.3855

21. Cloughesy TF, Cavenee WK, Mischel PS. Glioblastoma: from molecular pathology to targeted treatment. Annu Rev Pathol. 2014; 9:1-25. DOI: 10.1146/annurev-pathol-011110-130324 [PubMed: 23937436]

22. Sarkaria JN, Yang L, Grogan PT, Kitange GJ, Carlson BL, Schroeder MA, Galanis E, Giannini C, Wu W, Dinca EB, James CD. Identification of molecular characteristics correlated with glioblastoma sensitivity to EGFR kinase inhibition through use of an intracranial xenograft test panel. Mol Cancer Ther. 2007; 6:1167-1174. DOI: 10.1158/1535-7163.MCT-06-0691 [PubMed: 17363510]

23. Sasportas LS, Gambhir SS. Imaging circulating tumor cells in freely moving awake small animals using a miniaturized intravital microscope. PLoS One. 2014; 9:e86759.doi: 10.1371/journal.pone. 0086759 [PubMed: 24497977]

24. Slinker BK. The statistics of synergism. J Mol Cell Cardiol. 1998; 30:723-731. DOI: 10.1006/ jmcc.1998.0655 [PubMed: 9602421]

25. Kirson ED, Schneiderman RS, Dbaly V, Tovarys F, Vymazal J, Itzhaki A, Mordechovich D, Gurvich Z, Shmueli E, Goldsher D, Wasserman Y, Palti Y. Chemotherapeutic treatment efficacy and sensitivity are increased by adjuvant alternating electric fields (TTFields). BMC Med Phys. 2009; 9:1.doi: 10.1186/1756-6649-9-1 [PubMed: 19133110]

26. Kirson ED, Gurvich Z, Schneiderman R, Dekel E, Itzhaki A, Wasserman Y, Schatzberger R, Palti Y. Disruption of cancer cell replication by alternating electric fields. Cancer Res. 2004; 64:32883295. [PubMed: 15126372]

27. Clark PJ, Gaal J. Effects of Tumor Treating Fields(TTFields) and Temozolomide in MGMT Expressing and Non-Expressing Patient-derived Glioblastoma Cells. Neuro Oncology. 2016; 18:vi64.

28. Groves M, Schneiderman R, Zeevi E, Voloshin T, Giladi M, Kirson E, Weinberg U. Cytostatic Agents combined with Tumor Treating Fields (TTFields) in Glioma Cell Lines. Neuro Oncology. 2016; 18:vi133.

29. Giladi M, Schneiderman RS, Porat Y, Munster M, Itzhaki A, Mordechovich D, Cahal S, Kirson ED, Weinberg U, Palti Y. Mitotic disruption and reduced clonogenicity of pancreatic cancer cells in vitro and in vivo by tumor treating fields. Pancreatology : official journal of the International Association of Pancreatology. 2014; 14:54-63. DOI: 10.1016/j.pan.2013.11.009

30. Porat Y, Shteingauz Anna, Giladi M, Schneiderman R, Voloshin T, Munster M, Blat R, Kirson E, Weinberg U, Palti Y. Tumor Treating Fields (TTFields) induce Autophagy in Glioma Cells. Neuro Oncology. 2016; 18:vi65-66.

31. Kessler AF, Frömbling GE. Tumor Treating Field (TTField) effects on Glioblastoma Cells are augumented by Mitotic Checkpoint Inhibition. Neuro Oncology. 2016; 18:vi61.

32. O'Connell D, Shen V, Loudon W, Bota DA. First report of tumor treating fields use in combination with bevacizumab in a pediatric patient: a case report. CNS Oncol. 2016; doi: 10.2217/ cns-2016-0018

33. Hottinger AF, Stupp R, Homicsko K. Standards of care and novel approaches in the management of glioblastoma multiforme. Chin J Cancer. 2014; 33:32-39. DOI: 10.5732/cjc.013.10207 [PubMed: 24384238]

34. Kim EH, Kim YJ, Song HS, Jeong YK, Lee JY, Sung J, Yoo SH, Yoon M. Biological effect of an alternating electric field on cell proliferation and synergistic antimitotic effect in combination with ionizing radiation. Oncotarget. 2016; doi: 10.18632/oncotarget.11407

35. Dhami J, Chang E, Gambhir SS. Withaferin A and its potential role in glioblastoma (GBM). J Neurooncol. 2016; doi: 10.1007/s11060-016-2303-x

36. Santagata S, Xu Y-m, Wijeratne EMK, Kontnik R, Rooney C, Perley CC, Kwon H, Clardy J, Kesari S, Whitesell L, Lindquist S, Gunatilaka AAL. Using the Heat-Shock Response To Discover Anticancer Compounds that Target Protein Homeostasis. ACS Chem Biol. 2012; 7:340-349. DOI: 10.1021/cb200353m [PubMed: 22050377]

37. Sharada AC, Solomon FE, Devi PU. Toxicity of Withania somnifera root extract in rats and mice. IntJPharmacog. 2014; 3:205-212. 
38. Devi PU. Withania somnifera Dunal(Ashwagangha):Potential plant source of a promising dug for cancer chemotherapy and radiosensitization. Indian journal of experimental biology. 2014; 34:927-932.

39. Tiwari R, Chakraborty S, Saminathan M, Dhama K, Singh SV, et al. Ashwagandha (Withania somnifera): Role in Safeguading Health, Immunomodulatory Effects, Combating Infections and Therapeutic Application: A Review. Journal of Biological Sciences. 2014; 2:77-94.

40. Raut A, Rege N, Shirolkar S, Pandey S, Tadvi F, Solanki P, Vaidya R, Vaidya A, Kene K. Exploratory study to evaluate tolerability, safety, and activity of Ashwagandha (Withania somnifera) in healthy volunteers. Journal of Ayurveda and integrative medicine. 2012; 3:111.doi: 10.4103/0975-9476.100168 [PubMed: 23125505]

41. Borasi G, Nahum A, Paulides MM, Powathil G, Russo G, Fariselli L, Lamia D, Cirincione R, Forte GI, Borrazzo C, Caccia B, di Castro E, Pozzi S, Gilardi MC. Fast and high temperature hyperthermia coupled with radiotherapy as a possible new treatment for glioblastoma. J Ther Ultrasound. 2016; 4:32.doi: 10.1186/s40349-016-0078-3 [PubMed: 27980785]

42. Hodges TR, Ferguson SD, Heimberger AB. Immunotherapy in glioblastoma: emerging options in precision medicine. CNS Oncol. 2016; 5:175-186. DOI: 10.2217/cns-2016-0009 [PubMed: 27225028]

43. Gambhir L, Checker R, Sharma D, Thoh M, Patil A, Degani M, Gota V, Sandur SK. Thiol dependent NF-kappaB suppression and inhibition of T-cell mediated adaptive immune responses by a naturally occurring steroidal lactone Withaferin A. Toxicol Appl Pharmacol. 2015; 289:297312. DOI: 10.1016/j.taap.2015.09.014 [PubMed: 26408225]

44. Diamant G, Ram Z, Volovitz I. Evaluating the in-vitro Effects of Tumor-Treating Fields on T-Cell Responses. Neuro Oncology. 2016; 18:vi93.

45. Holtzman T. Tumor Treating Fields exposure of Tumor Cells induce activation phenotype in Immune Cell. Neuro Oncology. 2016; 18:vi92. 


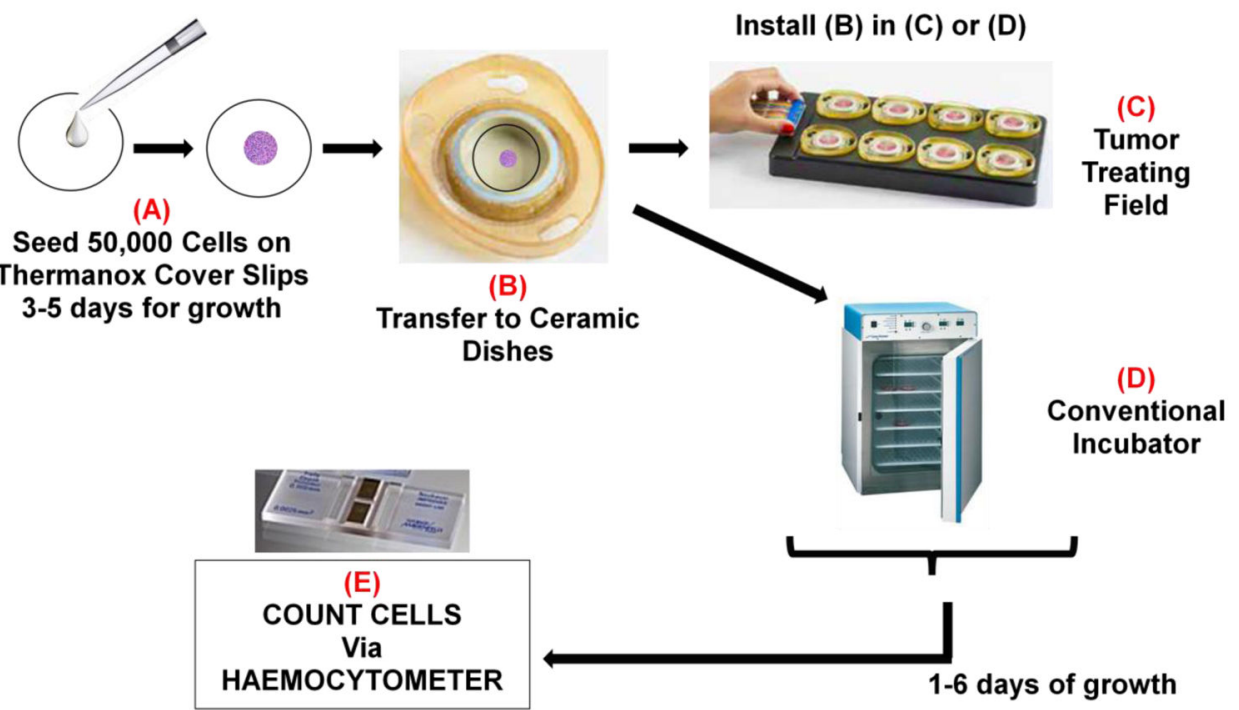

Figure 1.

Schematic of a standard TTField experiment. (A) Unless mentioned otherwise, 50,000 single cells were suspended in $200 \mu \mathrm{L}$ of media and seeded in the middle of a $22 \mathrm{~mm}$ diameter cover slip. The cover slips were placed in a 6-well plate and allowed to incubate in a conventional tissue culture incubator $\left(37^{\circ} \mathrm{C}, 95 \%\right.$ air, $\left.5 \% \mathrm{CO}_{2}\right)$ overnight. Once cells adhered to the cover slip, an additional $2 \mathrm{~mL}$ of media was added to each well. The cells remained on the cover slips for 2-3 days in order to achieve the growth phase, before they were transferred to (B) the wells of an inovitro ${ }^{\mathrm{TM}}$ TTField system ceramic dish. The dishes were pre-filled with $2 \mathrm{~mL}$ of media. The ceramic dishes were placed either in (C) base plates that are connected to the power box of the inovitro ${ }^{\mathrm{TM}}$ TTField system and then into a special incubator for the alternating electric current conditions or (D) into a conventional tissue culture incubator $\left(37^{\circ} \mathrm{C}, 95 \%\right.$ air, $\left.5 \% \mathrm{CO}_{2}\right)$ for the control samples. The cells were grown for 3-6 days with daily changes of media in both the TTField and control conditions. (E) At the end of this time, the cover slips were placed in 6-well plates and the cells processed for cell counting or for bioluminescence imaging. 

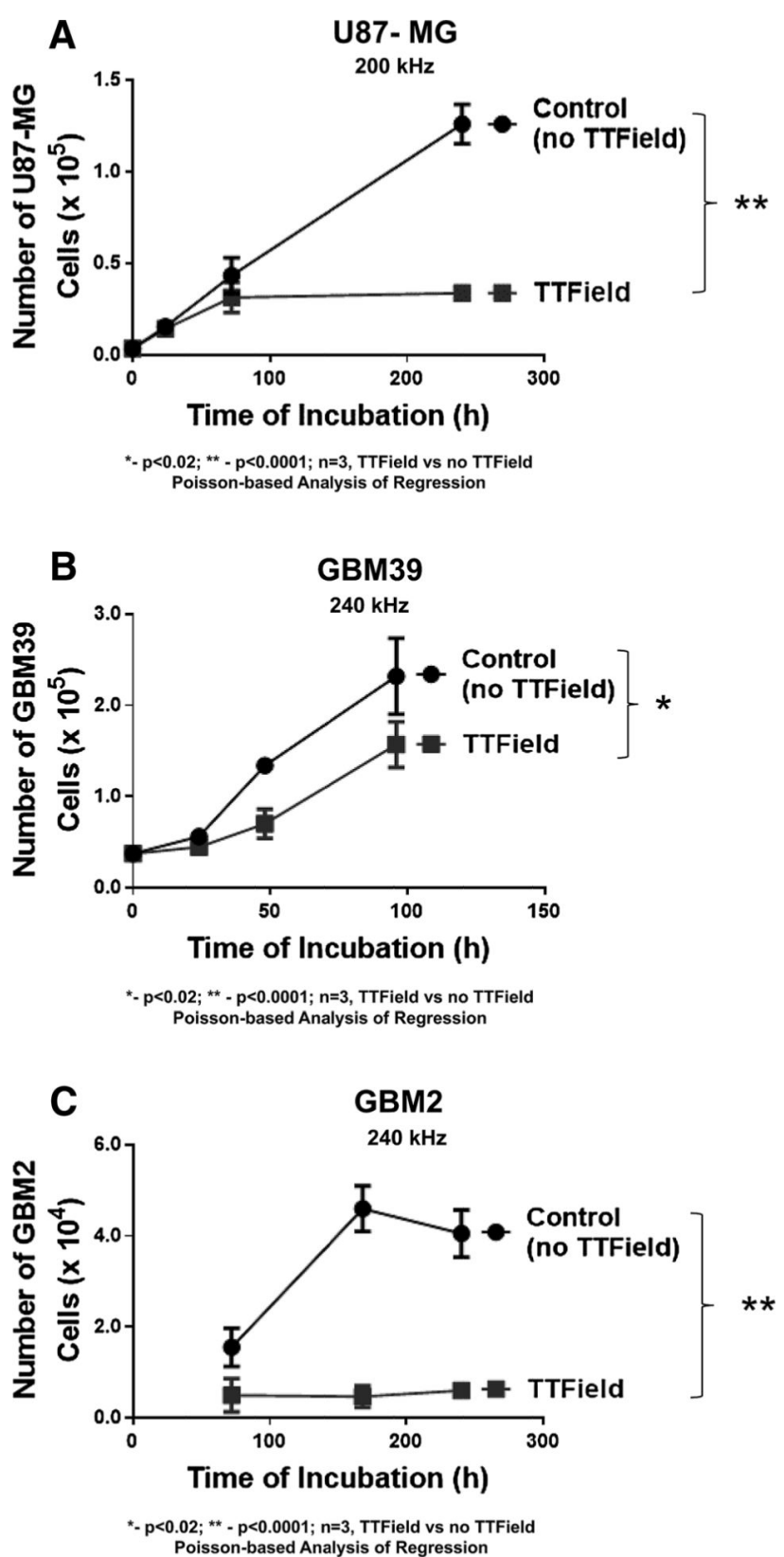

Figure 2.

Time course in the proliferation of human GBM cells (A) U87-MG, (B) GBM39, and (C) GBM2 in the presence of intermediate range (200-240 kHz) TTFields (black line, filled square data points) or under no TTField (black lines, filled round data points). Strength of electric field estimated at $4 \mathrm{~V} / \mathrm{cm}$. For all subfigures, $\mathrm{N}=3$ samples per data point, $*$ indicates significant difference $(\mathrm{p}<0.02)$ between TTField vs. non-TTField, while $* *$ indicates significant difference of $\mathrm{p}<0.0001$ between TTField vs. non-TTField and error bars represent standard deviation of four cell count measurements per three samples per condition. Except for GBM39 shown in 2B all samples were initially seeded at 50,000 cells/ cover slip. GBM39 were seeded at 10,000 cells/cover slip. 
(A)

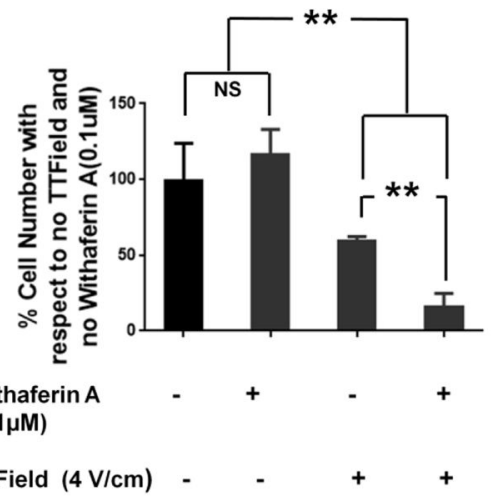

(B)

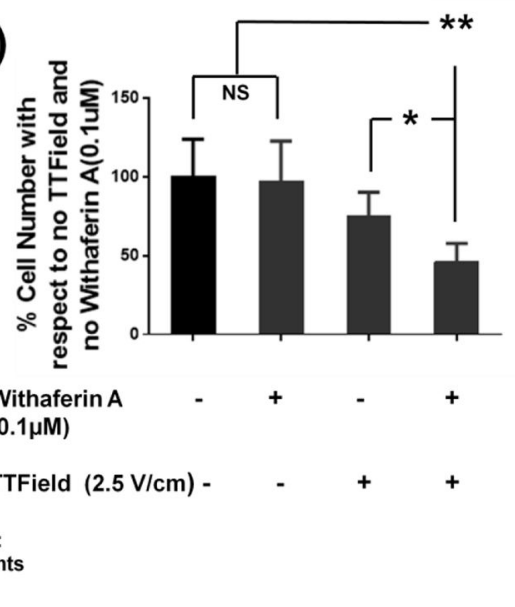

\section{U87-MG}

(C)

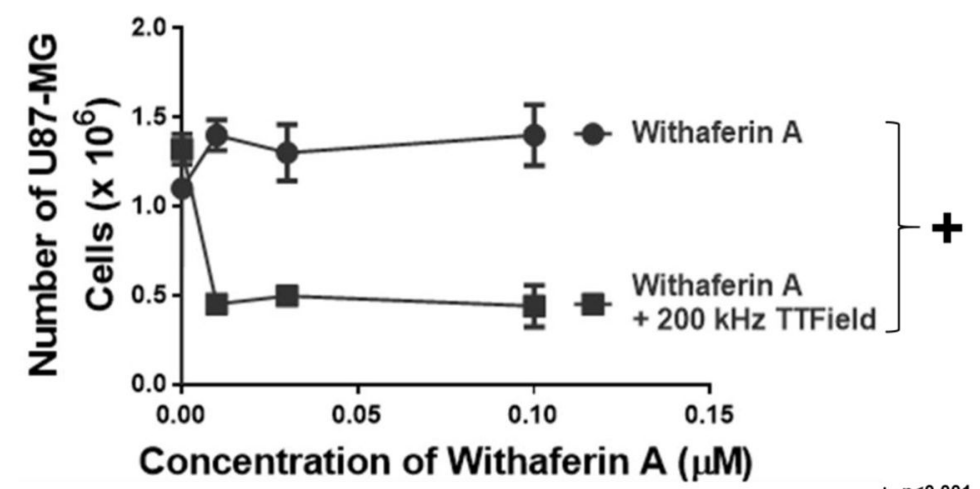

Poisson-based Analysis of Regresssion $n=3$ measurements per condition Withaferin A vs. TTField+Withaferin A 


\section{GBM39}

(D)

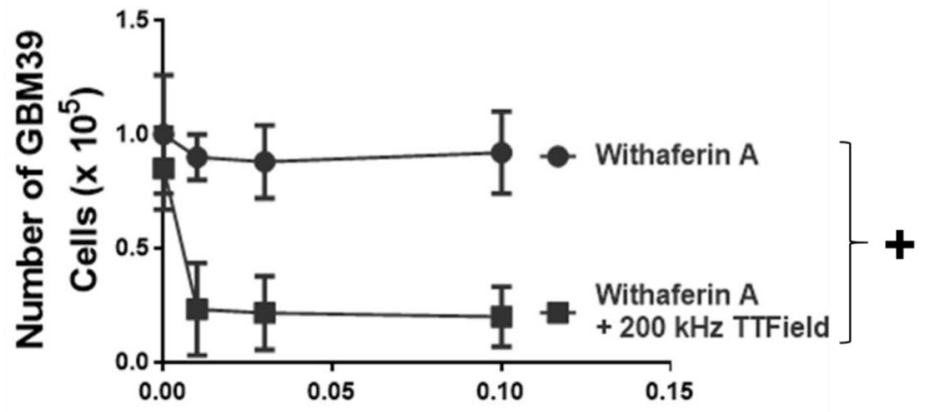

Concentration of Withaferin $\mathrm{A}(\mu \mathrm{M})$

$+-p<0.001$
Poisson-based Analysis of Regresssion $n=3$ measurements per condition Withaferin A vs. TTField+Withaferin A

GBM2

(E)

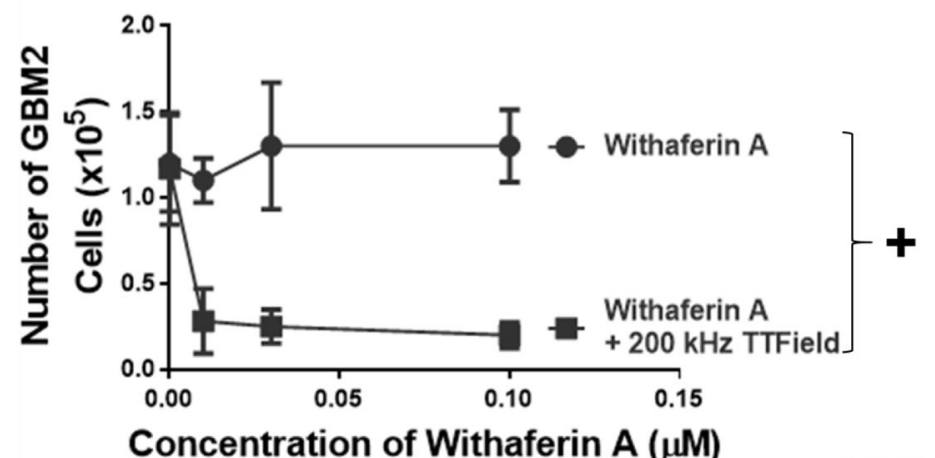

Poisson-based Analysis of Regresssion $\mathrm{n}=3$ measurements per condition

3F

No

$0.1 \mu \mathrm{M}$

Withaferin A Withaferin A

No
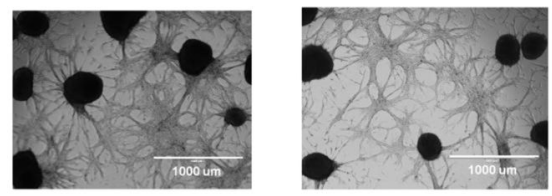

TTField $(4 \mathrm{~V} / \mathrm{cm})$
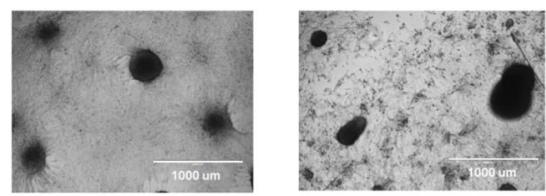

4X Magnification

Figure 3. 
Effect of treatments of $0.1 \mu \mathrm{M}$ Withaferin A, (A) $4 \mathrm{~V} / \mathrm{cm}$ or (B) $2.5 \mathrm{~V} / \mathrm{cm}$, TTFields (200 $\mathrm{kHz}$ ), neither, or both on U87-MG cells. $\mathrm{N}=3$ samples per condition (12 measurements in all). Counts were taken either (A) 3 days or (B) 6 days after treatment. ** indicates significant difference of $p<0.0001$ while * represents significant difference of $p<0.02$ for all 2-way ANOVA comparisons (i.e. Figure 3A and 3B). Error bars represent standard deviation of four cell count measurement per three samples per condition. (C-E) Dose response to Withaferin A ( 0 to $0.1 \mu \mathrm{M}$ or 0 to $0.3 \mu \mathrm{M}$ ) by human glioma cells (C) U87-MG, (D) GBM39, and (E) GBM2 as assessed by cell counting in the presence of $200 \mathrm{kHz}$ TTFields (black line, closed squares) or in its absence (black line, closed circles). (F) Pictographs of U87-MG under no treatment, single treatments of Withaferin A $(0.1 \mu \mathrm{M})$ or $200 \mathrm{kHz}$ TTFields, or both (all pictures taken 6 days after start of treatment). White bars represent distance of $1000 \mu \mathrm{m}$. For all subfigures, + indicates significant difference ( $\mathrm{p}<0.001)$ of plots as assessed by Poisson-based significance of regression analysis (test for synergy), and error bars represent standard deviation of four cell count measurement per three samples per condition. 


\section{A}
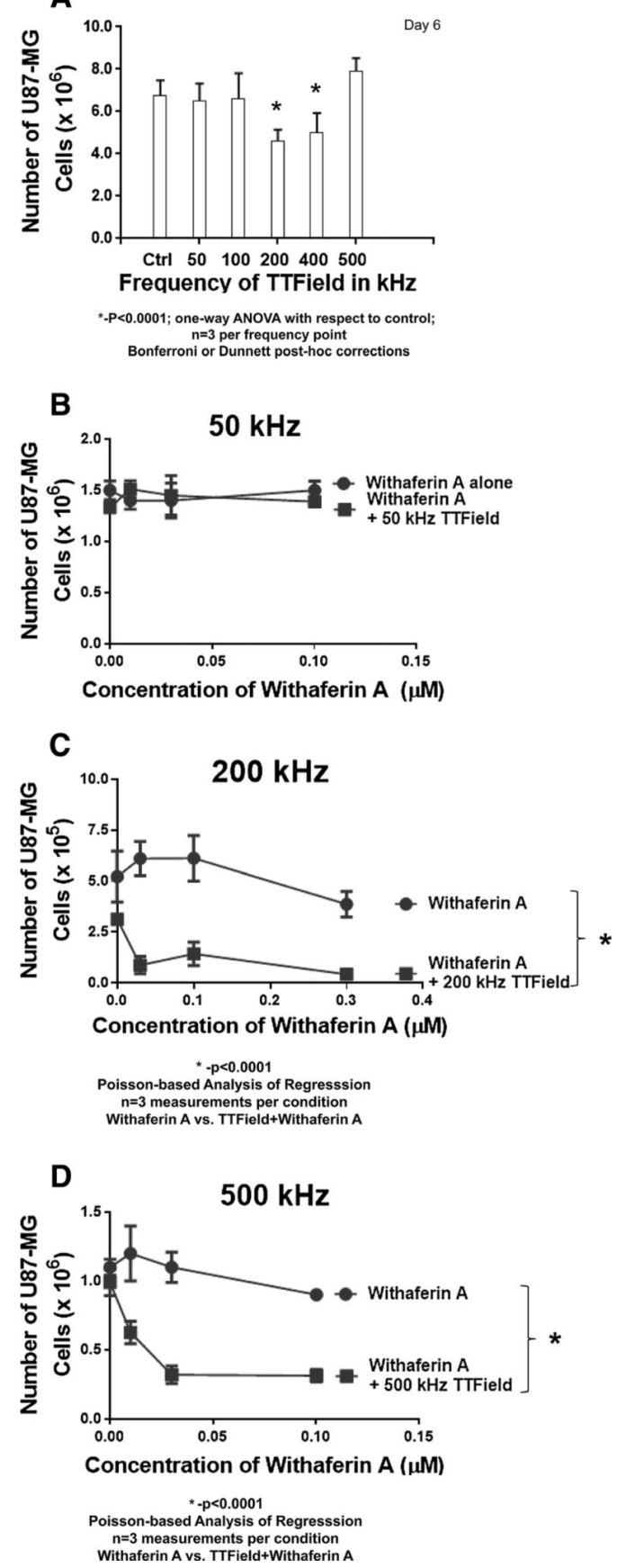

\section{Figure 4.}

(A) Comsol 4.2 modeling of the distribution of the alternating electric field in a ceramic dish from the inovitro ${ }^{\mathrm{TM}}$ apparatus that houses the cell-containing Thermanox ${ }^{\mathrm{TM}}$ coverslips. (B) Pictograph showing locations (center and edge) at which temperatures were recorded in the well of ceramic dishes with the use of a thermo-coupled temperature probe, and both the individual temperature measurements as well as the average outcomes (expressed in terms of ${ }^{\circ} \mathrm{C}$ ) for all ceramic dishes are displayed. Differences in measured temperature between center and edge was significantly different ( $\mathrm{p}<0.00001$, unpaired Student's t-test). (C) Time 
course of cell number increase for U87-MG cells under incubation temperatures of $37^{\circ} \mathrm{C}$ (black line, closed squares) and $39^{\circ} \mathrm{C}$ (black line, closed circles) showing no differences. 